\title{
Preliminary Three-Dimensional Volumetric Analysis of New Technique of Open Septal Reduction using Polydioxanone Plates for Nasoseptal Fracture
}

\author{
Kyubeom Kim ${ }^{1}$, Junhyung $\mathrm{Kim}^{1}$, Insik Yun ${ }^{2}$, Taehee $\mathrm{Jo}^{1}$, Jaehoon Choi ${ }^{1}$, Youngmin Shin $^{1}$, \\ and Woonhyeok Jeong ${ }^{1}$ \\ ${ }^{1}$ Keimyung University Dongsan Medical Center \\ ${ }^{2}$ Gangnam Severance Hospital
}

November 28, 2020

\begin{abstract}
Objectives: In this study, we designed a new technique for open septal reduction using a polydioxanone (PDS) plate and compared it with closed reduction. Design, Setting, Participants: This study included nineteen consecutive patients with nasoseptal fracture: ten receiving open reduction with a PDS plate (PDS group) and nine undergoing closed reduction (CR group). Open septal reduction was performed after closed reduction for nasal bone fracture. A mucoperichondrial flap was unilaterally elevated, and the deviated septal cartilage was reduced. The PDS plate was inserted horizontally above the vomerine suture. Surgical outcome was analyzed with three-dimensional volumetry and with a quality-of-life scale for nasal obstruction (NOSE scale). Results: Complications included one case of septal perforation in the CR group and one case of PDS exposure and septal hematoma in the PDS group. In the 3D volumetric analysis of the PDS group, the median value of the nasal cavity change significantly differed between $1.14 \mathrm{~mL}$ (interquartile range; 0.46 to 2.4 ) at the preoperative CT scan and 0.33 $\mathrm{mL}$ (interquartile range; -0.22 to 1.29$)$ at the postoperative $\mathrm{CT}$ scan $\left({ }^{* *} \mathrm{p}=0.0039\right)$. The NOSE scale revealed significant improvement in nasal obstruction postsurgically (median value, 42.5 to $7.5 ;{ }^{*} \mathrm{p}=0.0139$ ) in the PDS group. Conclusion: PDS plates potentially present a new concept of open septal reduction in terms of septal reinforcement compared with the subtractive approach of open septal reduction.
\end{abstract}

\section{ABSTRACT}

Objectives: In this study, we designed a new technique for open septal reduction using a polydioxanone (PDS) plate and compared it with closed reduction.

Design, Setting, Participants: This study included nineteen consecutive patients with nasoseptal fracture: ten receiving open reduction with a PDS plate (PDS group) and nine undergoing closed reduction (CR group). Open septal reduction was performed after closed reduction for nasal bone fracture. A mucoperichondrial flap was unilaterally elevated, and the deviated septal cartilage was reduced. The PDS plate was inserted horizontally above the vomerine suture. Surgical outcome was analyzed with three-dimensional volumetry and with a quality-of-life scale for nasal obstruction (NOSE scale).

Results: Complications included one case of septal perforation in the CR group and one case of PDS exposure and septal hematoma in the PDS group. In the 3D volumetric analysis of the PDS group, the median value of the nasal cavity change significantly differed between $1.14 \mathrm{~mL}$ (interquartile range; 0.46 to 2.4 ) at the preoperative $\mathrm{CT}$ scan and $0.33 \mathrm{~mL}$ (interquartile range; -0.22 to 1.29 ) at the postoperative CT scan $\left({ }^{* *} p=0.0039\right)$. The NOSE scale revealed significant improvement in nasal obstruction postsurgically (median value, 42.5 to $7.5 ;{ }^{*} p=0.0139$ ) in the PDS group. 
Conclusion: PDS plates potentially present a new concept of open septal reduction in terms of septal reinforcement compared with the subtractive approach of open septal reduction.

\section{Keypoints}

- This study is the first report of using a PDS plate for open septal reduction in nasoseptal fracture.

- We analyzed the effectiveness of our technique with quantitative data and standardized NOSE scale.

- PDS plate provides a structural support to straighten the septum for nasoseptal fracture.

- Open septal reduction using PDS plates is meaningfully technique in terms of septal reinforcement compared to traditional closed reduction.

\section{INTRODUCTION}

Nasal bone fracture is the most common fracture of the facial bone because of its prominent position at the center of the face. In 2018, nasal bone fracture was diagnosed in 49,878 patients according to data from the Korean National Health Insurance Service (NHIS). Closed and open reductions were performed in 18,972 and 1,232 patients, respectively. Likewise, in Korea, almost all nasal bone fractures are treated with closed reduction (CR) as in other countries. ${ }^{1}$ Nasal septal fractures frequently coincide with nasal bone fractures, which are reported with a frequency of up to $45 \%$ in computed tomography and $41 \%$ to $96 \%$ in intraoperative situations. ${ }^{2-4}$ Adequate septal management is important to prevent postoperative nasal obstruction and nasoseptal deformity. ${ }^{2}$

The initial management of septal fracture could be performed by a closed or an open approach. Open septal reduction has been performed with septoplasty or submucosal resection, which are removal techniques of deviated septal cartilage or perpendicular plates of ethmoid bone. ${ }^{2}$ Some authors have reported that open septal reduction could improve long-term esthetic and functional results in nasoseptal fracture. ${ }^{2,5-7}$ Nevertheless, there was hesitation to manage septal fracture aggressively because of the risk of destabilization of the nasal framework, septal perforation and increased difficulty when secondary septoplasty occurred. ${ }^{8}$ Therefore, we designed a new technique of open septal reduction using a polydioxanone plate (PDS) plate, which is more tissue preserving and minimally dissected than the traditional technique. Furthermore, we compared our method with the CR method by the objective method of three-dimensional volumetry and the patientreported functional outcome of the nasal obstruction scale.

\section{PATIENTS AND METHODS}

From June 2017 to December 2019, we reviewed nineteen consecutive patients with nasoseptal fractures who received surgery at our institution. We reviewed the data regarding patient demographics, surgical procedures, preoperative and postoperative computed tomography, the Nasal Obstruction Symptom Evaluation (NOSE) scale, and complications from our database. We used SPIRIT 2013 as a reporting guideline. The exclusion criteria were patients who had improper medical records and NOSE scores and the absence of preoperative and minimum scores 6 months after postoperative computed tomography. We divided the patients into two groups: a closed reduction group (CR group) and a PDS plate group (PDS group). When nasal mucosal injury was observed, we performed closed reduction and those who did not have nasal mucosal injury underwent open septal reduction with a PDS plate. This study was approved by the Keimyung University Dongsan Hospital Institutional Review Board (2020-03-038).

\section{Surgical Technique}

The procedure was performed with general anesthesia in all patients. First, CR was performed to correct nasal bone fracture using a blade handle and Walsham forceps. Then, open septal reduction was performed in the PDS group. Open septal reduction was performed through a hemitransfixion incision. Before the incision was made, we checked for any tears of the nasal mucosa on both sides of the nasal septum using nasal speculum or nasal endoscopy. If a tear was detected on one side, the incision was made on the other side of the nasal septum. If there were no tears on either side of the nasal mucosa, we preferred to make the incision on the concave side. After the incision was made, a mucoperichondrial flap was unilaterally elevated, and the deviated septal cartilage was reduced using a Freer elevator. In the PDS group, the 0.25-mm-thick 
PDS plate was cut to about $2 \mathrm{~cm}$-height with scissors. In the last 7 patients, we made holes in the PDS plate using a 1-mm punch biopsy device. The PDS plate was placed beside the fractured septal cartilage horizontally above the vomerine suture (Fig. 1). The PDS plate was not inserted diagonally close to the dorsal septum to avoid narrowing of the internal valve due to the thickness of the PDS plate. After insertion of the PDS plate, it was fixed with 3-0 Chromic mattress sutures through the full layers of the septum. The incision was closed with 4-0 Chromic suture. Nasal packing was performed on both nostrils with Merocel ${ }^{\circledR}$ (Medtronic Xomed, FL, USA). In the control group, closed septal reduction was performed. Closed septal reduction was performed following the traditional method using Ash forceps and a blade handle. After reduction, a soft silicone sheet was inserted into both nostrils to support the septum and fixed with a 3-0 Chromic mattress suture through the full layers of the septum. The silicone sheet was removed 5 to 7 days after the operation.

\section{Three-Dimensional Volumetric Analysis for the Nasal Cavity}

The nasal cavity was bounded by the nasal bones anteriorly, the posterior edge of the septal cartilage posteriorly, the nasal conchae laterally, the cribriform plate of the ethmoidal bone superiorly, and the palatal processes of the maxilla and horizontal portion of the palatine bone inferiorly. Volumetric measurements were calculated for each patient's nasal cavity containing septal cartilage based on the DICOM files using Invivo $6^{\circledR}$ software (Anatomage, CA, USA). Each CT slice was cut to a 5 -mm thickness axially by the program. Nasal cavity margins were marked with the program tool called 'Freehand Sculpture' on the axial view. After marking the nasal cavity margins on every slice cut ranging from the superior to inferior boundary, the whole volume of the nasal cavity was measured. Then, the right side of the nasal cavity was also measured based on the deviated septal cartilage in the same manner. The left side volume was calculated through the differences between the whole volume and the right side volume. The difference between the two sides of the nasal cavity was calculated and compared on preoperative and postoperative CT scans (Fig. 2).

\section{Evaluation of Nasal Obstruction}

The NOSE scale translated into Korean was used to assess nasal obstruction symptoms preoperatively and postoperatively. The obstruction-related items contained 5 items, and all items were scored using a 5-point Likert scale from 0 to 4 (not a problem, very mild problem, moderate problem, fairly bad problem, and severe problem). The patients had to assess their difficulties with breathing through the nose in general with a range from none to severe. The sum of five scores was multiplied by 5 to obtain a final score ranging from 0 to 100. All patients were asked to complete the NOSE scale preoperatively and a minimum of 3 months after surgery (Supplementary data 1).

\section{Statistical Analysis}

Statistical analyses were performed by the Wilcoxon signed rank test using GraphPad Prism $5^{\circledR}$ (GraphPad Software, Inc., CA, USA). Statistical data are presented as the median value with interquartile range. Values of $p<0.05$ were considered statistically significant.

\section{RESULTS}

Nineteen consecutive patients were included in this study. The CR group consisted of 9 patients: 7 males and 2 females. The PDS group consisted of 10 patients: 8 males and 2 females. The mean ages of the CR group and PDS group were 41 and 43.3 years, respectively. The type of nasal bone fracture in the CR group was comminuted fracture in 6 patients and simple fracture in 3 patients. The type of nasal bone fracture in the PDS group was comminuted fracture in 8 patients and simple fracture in 2 patients. One case of septal perforation was noted as a postoperative complication in the CR group. The patient had a comminuted septal fracture and mucoperiosteal tearing at initial injury. One case of septal hematoma was noted 3 months after surgery in the PDS group. Septal hematoma was resolved by needle aspiration and nasal packing. Although the PDS plate was exposed 4 months after surgery in one case, spontaneous healing without septal perforation and with simple removal of the exposed PDS plate was observed (Fig. 3).

In the 3D volumetric analysis, the difference in both nasal cavities was compared between preoperative and 
postoperative values (Fig. 4). The preoperative volume difference between the CR group and the PDS group was not statistically different. (1.6 mL versus $1.14 \mathrm{~mL} ; p>0.4482)$ In the $\mathrm{CR}$ group, the median value of the difference in the nasal cavity did not differ: $1.6 \mathrm{~mL}$ (interquartile range; 0.71 to 2.89 ) at the preoperative $\mathrm{CT}$ scan and $1.01 \mathrm{~mL}$ (interquartile range; 0.49 to 1.86$)$ at the postoperative CT scan $(p>0.05)$. However, in the PDS group, the median value of the difference in the nasal cavity was significantly different: $1.14 \mathrm{~mL}$ (interquartile range; 0.46 to 2.4 ) at the preoperative $\mathrm{CT}$ scan and $0.33 \mathrm{~mL}$ (interquartile range; -0.22 to 1.29 ) at the postoperative CT $\operatorname{scan}\left({ }^{* *} p=0.0039\right)$.

The NOSE scale was employed to analyze the change in nasal obstruction (Fig. 5). The preoperative NOSE scale between the CR group and the PDS group was not statistically different. (the value of 20 versus the value of $42.5 ; p>0.0966)$ In the CR group, the median score on the NOSE scale did not differ between the value of 20 (interquartile range: 5 to 35) before surgery and the value of 15 (interquartile range: 7.5 to 22.5) after surgery $(p>0.05)$. However, in the PDS group, the median score on the NOSE scale was significantly different, namely, 42.5 (interquartile range; 18.7 to 71.25 ) before surgery and 7.5 (interquartile range; 0 to 15) after surgery $\left({ }^{*} p=0.0139\right)$.

\section{DISCUSSION}

In this study, we designed a new technique for open reduction of nasoseptal fracture using a PDS plate. The statistical analysis showed no difference of preoperative CT volume and NOSE scale between two groups. However, postoperative CT volume difference and NOSE scale significantly decreased in the PDS group. Our results show that compared with closed septal reduction, open septal reduction using a PDS plate provides better structural support to straighten the septum and improve nasal obstructive symptoms after surgery. (Fig. 6 and Supplementary 2)

In nasoseptal fracture, there is an accumulating basis of open septal reduction to improve treatment outcomes. Rohrich and Adams ${ }^{9}$ advocate adequate assessment and reconstruction of the nasal septum and nasal bone to reduce the secondary revision rate. Indeed, compared with closed septal reduction, open septal reduction dramatically reduced the residual septal deviation rate by $60 \%$ to $12.5 \%{ }^{10}$ Other studies have also reported that open septal reduction reduces the remaining septal deviation and nasal obstruction. ${ }^{2,6}$ Traditionally, open septal reduction has been performed by eliminating the deviated septum or perpendicular plate by septoplasty or submucosal resection (SMR). ${ }^{2,6,10}$ Septoplasty is a tissue-sparing technique in which there is limited removal of a deviated septum compared with SMR, for which complete excision of septal cartilage except 1 to $1.5 \mathrm{~cm}$ width L-strut is performed. Nevertheless, septoplasty inevitably increases mucosal damage, septal perforation, and the risk of destabilization of the nasal framework because the bilateral mucoperichondrial flap should be raised. Investigations of complications due to open septal reduction itself have been conducted, with one author reporting that $5.5 \%$ of nasal dorsal irregularities and $1.1 \%$ of septal perforations were postoperative complications. ${ }^{6}$ While traditional open septal reduction could weaken the septal framework by removing part of the septum, our technique was performed as reinforcement by a PDS plate without removal of the septum. In particular, we did not perform bilateral elevation of the mucoperiosteal flap.

Preservation of contact between the mucoperiosteal flap and septum might prevent absorption of septal cartilage by ischemia arising from bilateral vascular blockage. Furthermore, unilateral elevation of the mucoperiosteal flap might reduce the risk of penetrating injury of bilateral mucoperiosteal injury and the risk of septal perforation. However, we experienced two cases of complications that arose from PDS plate insertion, including one case of delayed septal hematoma and one case of exposure of the PDS plate. For the third case of our technique, we used a 0.25-mm PDS plate without penetration. In the third case, the patient demonstrated septal hematoma at the three-month follow-up. Septal hematoma was aspirated with septal packing for 5 days and resolved without further complications. After this case, we made holes on the PDS plate using a 1-mm punch biopsy device and had never experienced this situation again. Exposure of the PDS plate developed in one patient who had the PDS plate inserted to the convex side of septal deviation due to mucosal tearing on the concave side. We preferred to insert a PDS plate on the concave side of septal deviation following the theory of unilateral spreader graft. In addition, mucoperichondrial elevation on the 
convexity of septal deviation might increase the chance of iatrogenic tearing of the mucoperichondrial flap by slipping down the Cottle elevator, which moves toward the mucoperiosteal flap. In particular, mucosal tearing could easily develop below the peak of convexity. However, this patient had mucosal tearing at the concave side, and we inserted a PDS plate at the convex side. We supposed the cause of PDS exposure was that the pushing force of convexity of the septal deviation and memory of the native tissue caused irritation of the mucoperichondrial flap by the PDS plate. The exposure of the PDS plate developed 4 months after surgery and was easily removed in the clinic because the PDS plate was already weakened by absorption. The patient had no signs of infection or sepal perforation and recovered by conservative management.

The FDA approved the application of the PDS plate for septorhinoplasty in 2010. The PDS plate demonstrated the initiation of absorption from 10 weeks and lost its continuity with partial degradation 15 weeks after implantation. ${ }^{11}$ After 25 weeks, the PDS plate was completely absorbed through hydrolysis with minimal fibrous scar tissue and newly formed cartilage in artificial cartilage defects. ${ }^{11}$ Several authors have reported favorable outcomes of PDS plates in septorhinoplasty. ${ }^{12-15}$ They used a PDS plate as a platform sutured with cartilaginous fragments to reinforce and straighten the grafts. Likewise, we hypothesized that the insertion of a PDS plate under a mucoperichondrial flap would be effective to support a broken septum by a long-lasting "stent" in septal fracture. PDS plates are available in three types based on thickness: $0.15 \mathrm{~mm}, 0.25 \mathrm{~mm}$, and $0.5 \mathrm{~mm}$ thicknesses. Most surgeons used the perforated type of 0.15 -mm PDS plate because the $0.25-\mathrm{mm}$ PDS plate induces vascular compromise due to its nonperforated nature. ${ }^{16}$ However, we determined that the 0.15-mm PDS plate reinforces the fractured septum because it is very thin and flexible. In fact, there have been reports that 0.25 -mm PDS without perforation does not induce vascular compromise of the septum with one-sided insertion. ${ }^{15}$ Some authors have reported favorable results using a 0.25 -mm plate perforated by a 16 -gauge needle. ${ }^{17}$ We also used a perforated 0.25 -mm PDS plate and obtained effective reinforcement for the fractured septum.

To our knowledge, the current study is the first report of using a PDS plate for open septal reduction in nasoseptal fracture. Nevertheless, the small number of patients is a potential limitation of this study, and we could obtain favorable results for the use of PDS plates in open septal reduction by objective analysis of three-dimensional volumetry and standardized NOSE scale. A future study involving a large population with long-term follow-up will be able to advance our results.

\section{CONCLUSION}

PDS plates are effective stenting materials to reinforce the fractured septum in open septal reduction. When open septal reduction is performed using a PDS plate, it is important to prevent complications when the PDS plate is inserted to the concave side of septal deviation and is used with added perforations. In our experience, PDS plates potentially present a new concept of open septal reduction in terms of septal reinforcement compared with the subtractive manner of open septal reduction.

\section{The data availability statement}

The data that support the findings of this study are available from the corresponding author, Jeong WH, upon reasonable request.

\section{REFERENCES}

1. Watson DJ, Parker AJ, Slack RW, Griffiths MV. Local versus general anaesthetic in the management of the fractured nose. Clin Otolaryngol Allied Sci 1988;13 :491-4.

2. Rhee SC, Kim YK, Cha JH, Kang SR, Park HS. Septal fracture in simple nasal bone fracture. Plast Reconstr Surg 2004;113:45-52.

3. Kim JH, Lee JW, Park CH. Cosmetic rhinoseptoplasty in acute nasal bone fracture. Otolaryngol Head Neck Surg 2013;149:212-8.

4. Kim JE PH, Yoon CH, Kim HJ. Analysis of nasal septal fracture combined in nasal bone fracture using computerized tomography. J Korean Soc Plast Reconstr Surg 1998;25 :852 - 9. 
5. Mondin V, Rinaldo A, Ferlito A. Management of nasal bone fractures.Am J Otolaryngol 2005;26 :181-5.

6. Andrades P, Pereira N, Borel C, Rocha L, Hernandez R, Villalobos R. A new approach to nasoseptal fractures: Submucosal endoscopically assisted septoplasty and closed nasal reduction. J Craniomaxillofac Surg 2016;44:1635-40.

7. Younes A, Elzayat S. The role of septoplasty in the management of nasal septum fracture: a randomized quality of life study. Int J Oral Maxillofac Surg 2016;45:1430-4.

8. Hoffmann JF. An Algorithm for the Initial Management of Nasal Trauma.Facial Plast Surg 2015;31 :183-93.

9. Rohrich RJ, Adams WP, Jr. Nasal fracture management: minimizing secondary nasal deformities. Plast Reconstr Surg2000;106:266-73.

10. DeFatta RJ, Ducic Y, Adelson RT, Sabatini PR. Comparison of closed reduction alone versus primary open repair of acute nasoseptal fractures. J Otolaryngol Head Neck Surg 2008;37 :502-6.

11. Boenisch M, Mink A. Clinical and histological results of septoplasty with a resorbable implant. Arch Otolaryngol Head Neck Surg2000;126 :1373-7.

12. James SE, Kelly MH. Cartilage recycling in rhinoplasty: polydioxanone foil as an absorbable biomechanical scaffold. Plast Reconstr Surg 2008;122 :254-60.

13. Gerlinger I, Karasz T, Somogyvari K, Szanyi I, Rath G, Moricz P, Boenish M. Extracorporal septal reconstruction with polydioxanone foil. Clin Otolaryngol 2007;32 :465-70.

14. Gubisch W. Extracorporeal septoplasty for the markedly deviated septum. Arch Facial Plast Surg 2005;7 :218-26.

15. Fuller JC, Levesque PA, Lindsay RW. Polydioxanone plates are safe and effective for L-strut support in functional septorhinoplasty. Laryngoscope 2017;127:2725-30.

16. Tweedie DJ, Lo S, Rowe-Jones JM. Reconstruction of the nasal septum using perforated and unperforated polydioxanone foil. Arch Facial Plast Surg 2010;12 :106-13.

17. Caughlin BP, Been MJ, Rashan AR, Toriumi DM. The effect of polydioxanone absorbable plates in septorhinoplasty for stabilizing caudal septal extension grafts. JAMA Facial Plast Surg2015;17 :120-5.

\section{Figure Legends}

Fig. 1. Intraoperative view of open septal reduction using the PDS plate. (A) After the mucoperichondrial flap was elevated unilaterally without tearing, a septal fracture was noted above the vomerine groove. (B) The 0.25-mm-thick PDS plate was cut for insertion into the fracture site. (C) The PDS plate was inserted horizontally above the vomerine groove to support the fracture site.

Fig. 2. Three-dimensional reconstruction of the CT scan for volumetric analysis. (A) Threedimensional reconstruction of the preoperative nasal cavity in the PDS group. There was septal deviation to the left nasal cavity and a discrepancy in the nasal cavity volume. (C) Three-dimensional reconstruction of the 6-month postoperative nasal cavity in the PDS group. The volume discrepancy was decreased by septal straightening.

Fig. 3. (A) The PDS plate was exposed through the caudal septal region of the convex side of septal deviation at the 4-month follow-up. (B) The PDS plate was easily removed in the clinic. (C) The mucosal wound spontaneously recovered without septal perforation after 1 month.

Fig. 4. Three-dimensional volumetry. The mean difference in nasal cavity volume was significantly decreased after surgery in the PDS group $\left({ }^{* *} p=0.0039\right)$. 
Fig. 5. NOSE scale. In the PDS group, the patient's reported nasal obstructive symptoms were significantly improved after surgery $\left({ }^{*} p=0.0139\right)$.

Fig. 6. Case 1. A 32-year-old woman who had a nasoseptal fracture and received open septal reduction using a PDS plate. (A) Preoperative photography revealed an obvious nasal deviation to the left side. (B) Six months after the operation, the nasal deviation was straightened by CR of the nasal bone and open septal reduction using the PDS plate. (C) There was an obvious difference in nasal cavity volume between the two sides at preoperative 3D volumetry. (D) Volume discrepancy between the two sides of the nasal cavity was improved after surgery.

Supplementary data 1. Nasal Obstruction Symptom Evaluation (NOSE) scale.

Supplementary data 2. Case 2. A 59-year-old woman who experienced exposure of a PDS plate after it was used for open septal reduction. (A) Preoperative photography revealed nasal deviation to the right side and loss of dorsal height. (B) Although part of the PDS plate was exposed and removed, as presented in Fig. 3 , the nasal deviation and dorsal height were successfully recovered by CR of the nasal bone and open septal reduction using the PDS plate. (C) There were septal deviation and discrepancy between the two sides on preoperative $3 \mathrm{D}$ volumetry. (D) The septal deviation was corrected after surgery.
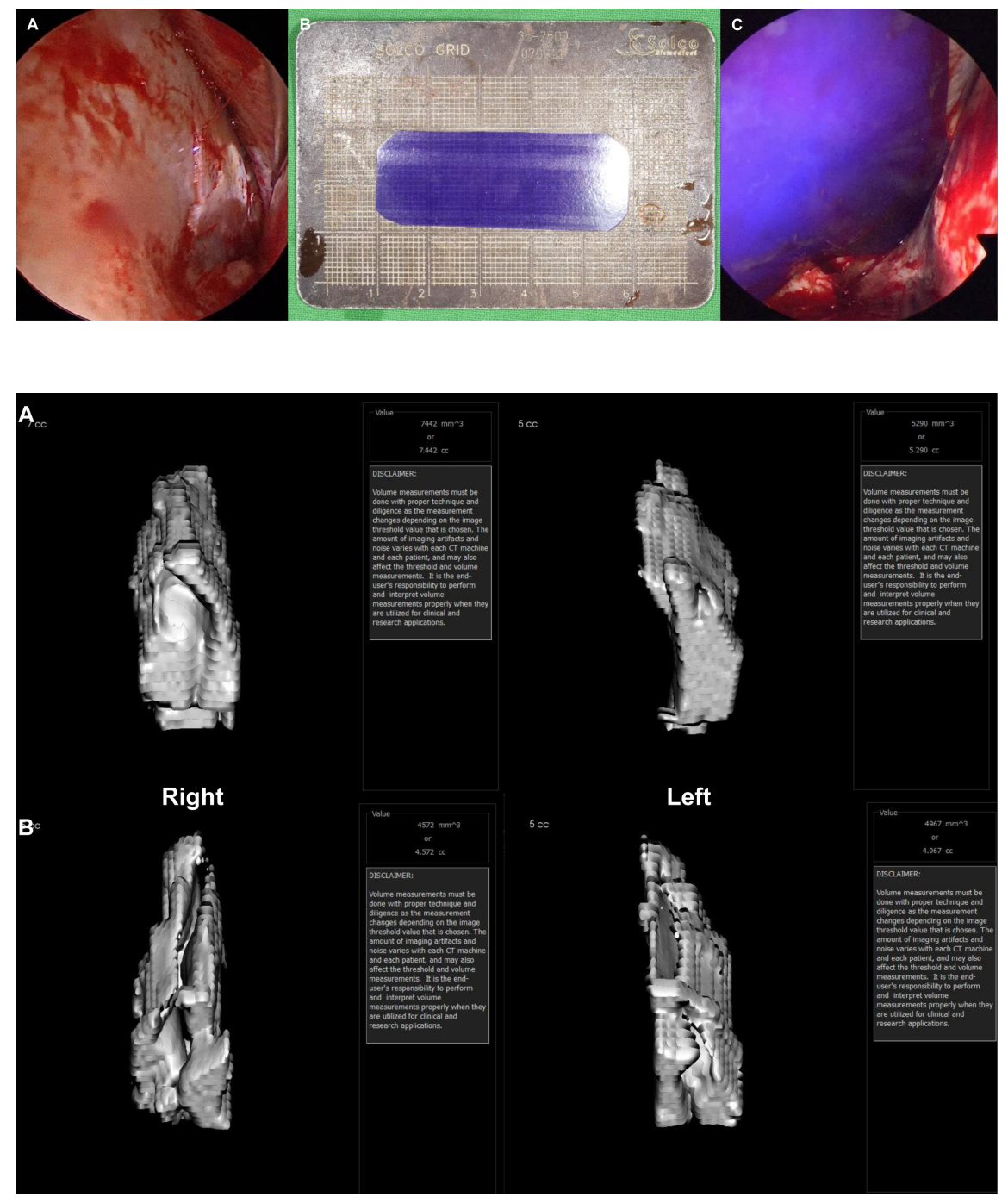

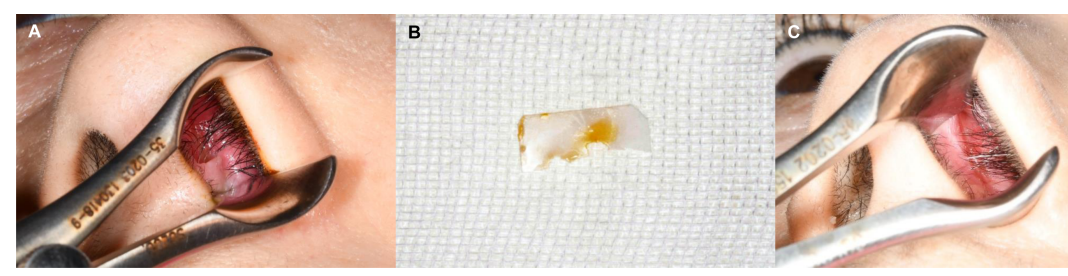

3D volumetry $(\mathrm{mL})$

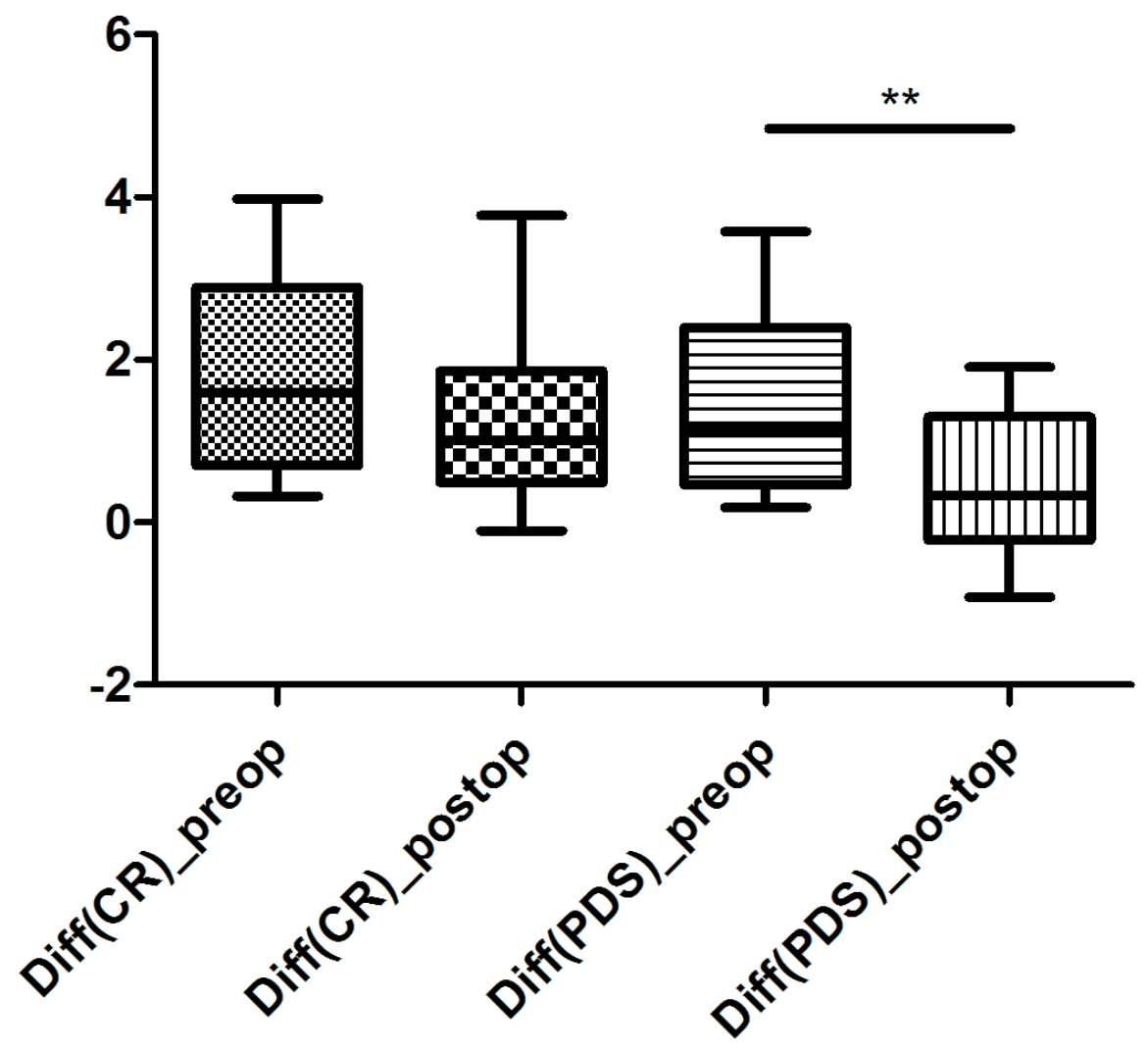




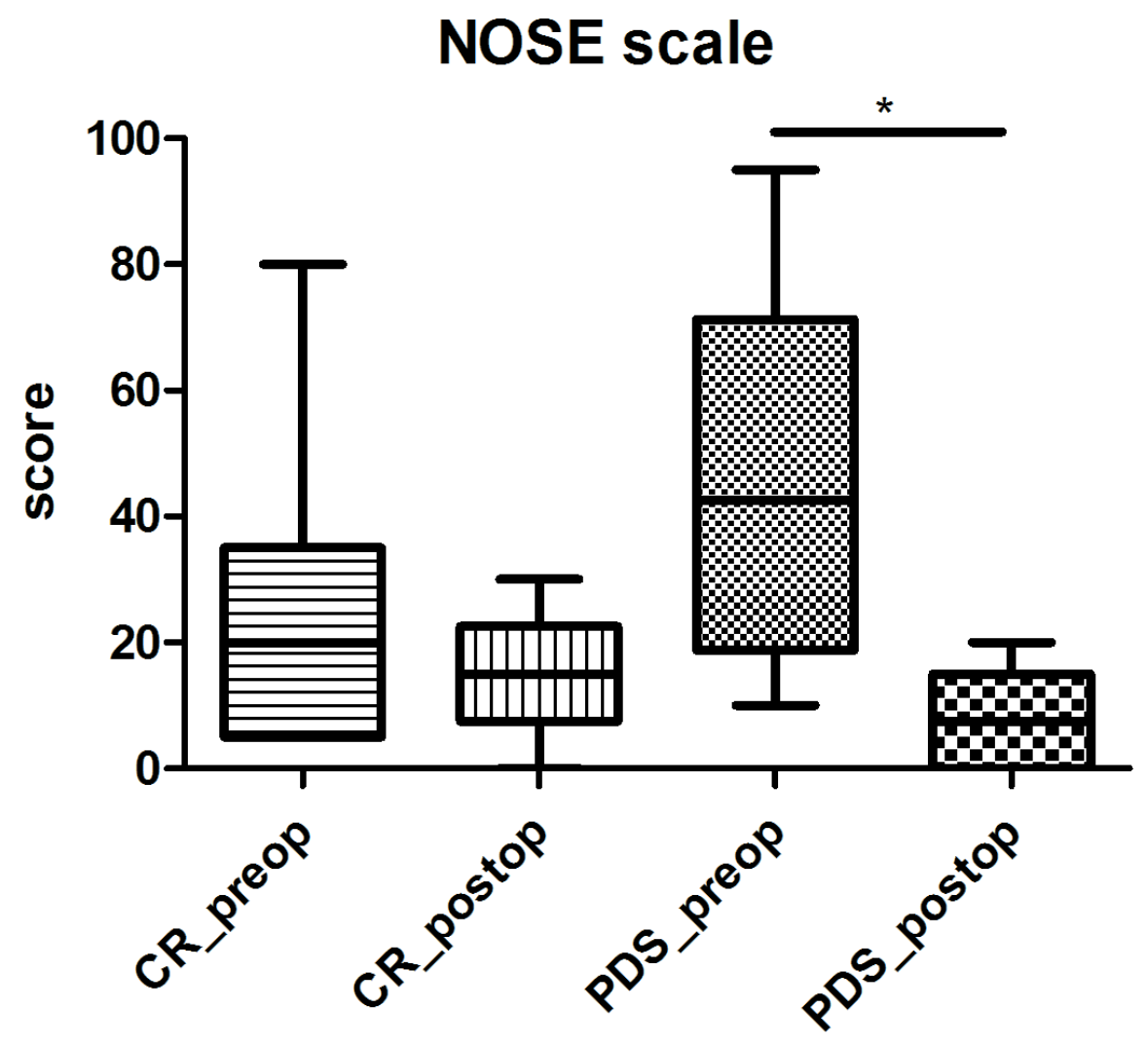




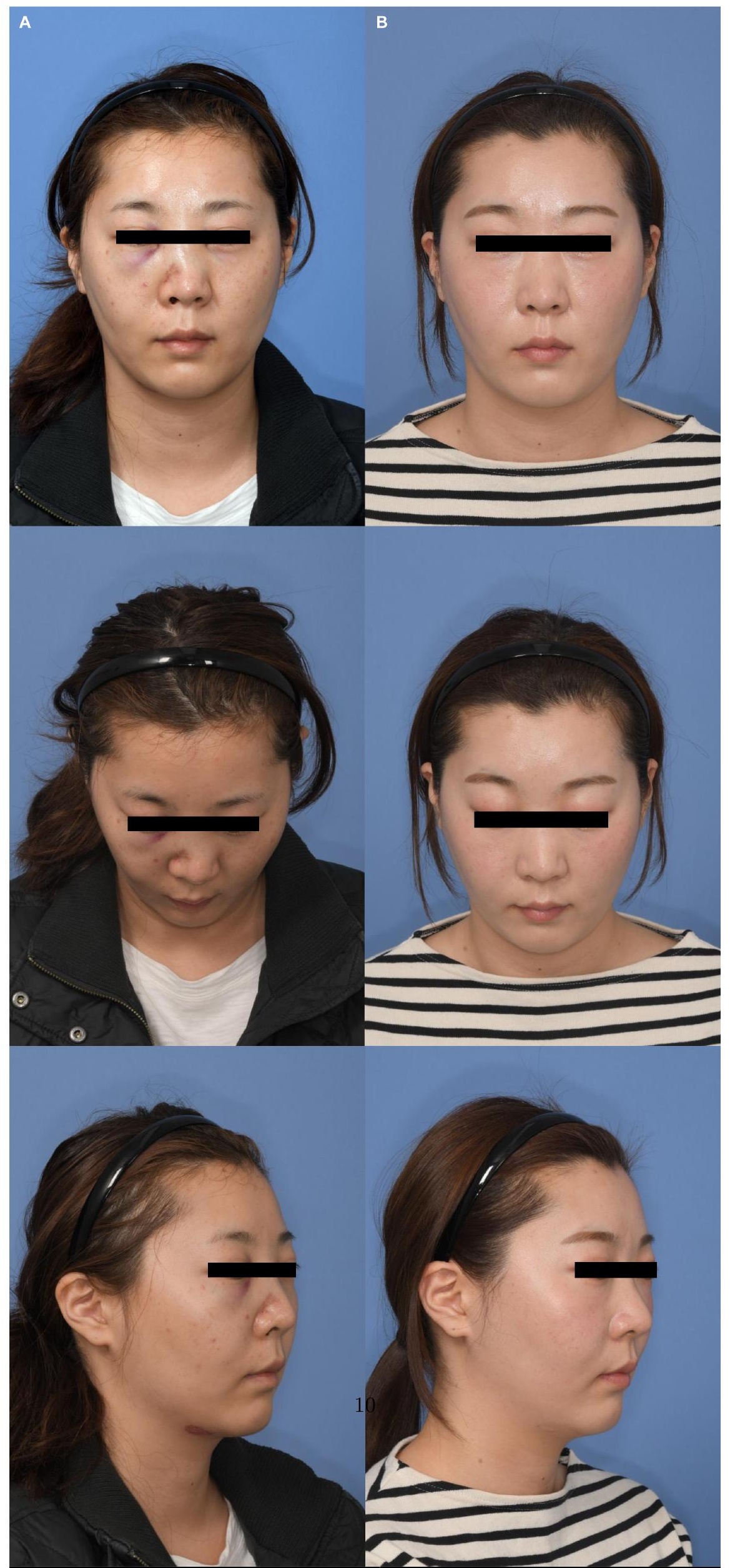

